\title{
Real-space multiple scattering theory for superconductors with impurities
}

\author{
Tom G. Saunderson $\odot,{ }^{1, *}$ Zsolt Győrgypál, ${ }^{2}$ James F. Annett $\odot,{ }^{1}$ Gábor Csire, ${ }^{3}$ Balázs Újfalussy $\odot,{ }^{2}$ and Martin Gradhand $\odot^{1}$ \\ ${ }^{1}$ HH Wills Physics Laboratory, University of Bristol, Tyndall Ave, BS8 1TL, United Kingdom \\ ${ }^{2}$ Institute for Solid State Physics and Optics, Wigner Research Centre for Physics, Hungarian Academy of Sciences, \\ P.O. Box 49, H-1525 Budapest, Hungary \\ ${ }^{3}$ Catalan Institute of Nanoscience and Nanotechnology (ICN2), CSIC, BIST, Campus UAB, Bellaterra, Barcelona 08193, Spain
}

(Received 18 September 2020; revised 18 November 2020; accepted 18 November 2020; published 4 December 2020)

\begin{abstract}
We implement the Bogoliubov-de Gennes (BdG) equation in real-space using the screened Korringa-KohnRostoker (KKR) method. This allows us to solve, self-consistently, the superconducting state for 3D crystals including substitutional impurities with a full normal-state DFT band structure. We apply the theoretical framework to bulk $\mathrm{Nb}$ with impurities. Without impurities, $\mathrm{Nb}$ has an anisotropic gap structure with two distinct peaks around the Fermi level. In the presence of nonmagnetic impurities, those peaks are broadened due to the scattering between the two bulk superconducting gaps, however the peaks remain separated. As a second example of self-consistent real-space solutions of the BdG equations, we examine superconducting clusters embedded within a nonsuperconducting bulk metallic host. This allows us to estimate the coherence length of the superconductor and we show that, within our framework, the coherence length of the superconductor is related to the inverse of the gap size, just as in bulk BCS theory.
\end{abstract}

DOI: 10.1103/PhysRevB.102.245106

\section{INTRODUCTION}

Inhomogeneities in superconductors have been of intense interest for many years. Impurities in bulk materials have been exploited to destroy superconductivity [1,2], create superconductivity by doping [3-5], determine the order parameter of superconductors [6-9], and create bound Yu-Shiba-Rusinov states in superconducting materials [10-14]. Furthermore, nanoscale structured superconducting materials have been engineered to provide artificial materials with desired characteristics, such as increased critical temperature $\mathrm{T}_{c}$, or granular as well as percolative superconductivity [15].

Modelling inhomogeneous systems such as these generally requires real-space solutions of the Bogoliubov-de Gennes (BdG) equations. Fetter [16] was one of the first to use localized models to describe nonmagnetic impurities in the superconducting state. It was shown that impurities in real materials will induce both spatial oscillations in the pairing potential $\Delta(r)$ and the electron density $\rho(r)$. In addition, the resonant enhancement of the scattering of quasiparticles with momentum near the Fermi momentum was identified. Later, Flatté and Byers [17] provided insightful models into how magnetic and nonmagnetic impurities behave in a free-electron s-wave superconducting medium. These models provided qualitative insight of a generic localized perturbation in a model superconductor, but lacked any quantitative predictive power to describe the complex impurity states which would occur in real materials. Material specific information about the superconducting gap variation on the Fermi surface were considered in realistic tight-binding models. $[6,8,18-$

\footnotetext{
*t.saunderson@bristol.ac.uk
}

23] In all those cases the impurities were used as a probe to investigate the superconducting gap structure and order parameter for unconventional superconductors. This quasiparticle interference on real surfaces has been visualized experimentally using scanning tunneling microscopy [24-33] and provided powerful insight into the superconducting state. Since most of the theoretical approaches work in reciprocal space direct comparison to experiments will typically involve Fourier transformations of the direct real-space analysis of the experiments.

Understanding such inhomogenous systems at the $a b$ initio level poses significant challenges, even for conventional electron-phonon BCS driven superconductors. In the bulk, modelling of phonon mediated s-wave superconductors has been successful using modern DFT techniques [34]. Incorporating impurities or nanoscale structured materials into these $a b$ initio methods would be possible in principle, but would become technically very challenging and computationally demanding. Even in bulk systems the full theory requires six-dimensional integrals both over the electron and phonon Brillouin zones, $\mathbf{k}$ and $\mathbf{Q}$. In systems without translational symmetry the corresponding real-space coupled electron-phonon equations would become significantly more difficult to solve. Similarly, for unconventional superconductors, models including impurities either use a simplified parametrization of the problem, or they are forced to use supercell approaches to incorporate the impurity site [35].

In this work, we will exploit the Korringa-Kohn-Rostoker (KKR) Greens function method which is ideally suited to treat the real-space impurity problem in a full quantitative $a b$ initio approach [36]. We combine the first-principles treatment of the impurity problem with the implementation of the Bogoliubov-de Gennes (BdG) equations, which we developed 
previously to describe superconductivity in periodic crystals and surfaces in $k$ space. [37,38] Within this framework a phenomenological parametrization of the pairing interaction introduces the parameter $\Lambda$, which is fixed by the experimentally found gap size. Such a treatment has been shown to reproduce experimentally observed gap anisotropies for various materials such as $\mathrm{Nb}, \mathrm{Pb}$, and $\mathrm{MgB}_{2}$ [38]. It has also been used to develop a quantitative theory for triplet pairing in $\mathrm{LaNiC}_{2}$ and $\mathrm{LaNiGa}_{2}[39,40]$.

This method incorporates the full orbital character of real impurities in contrast to previous tight-binding models. The explicit real-space description will allow for more direct comparison to local experimental probes having direct access to the local density of states (LDOS). After a brief introduction on the specific implementation in Sec. II, the method will be tested with a range of impurities in $\mathrm{Nb}$ in order to explore the different effects of impurities showing distinct orbital character in Sec. III. In Sec. IV, we apply this method to granular superconductors, solving ab initio a nanoscale superconducting cluster embedded in a normal metallic environment. We can view this system as a sort of inverse problem to that of an impurity in a bulk superconducting host. Solving the realspace BdG equations for the superconducting cluster allows us to make direct contact to the concept of the superconducting coherence length as applied to granular superconductivity. After Sec. IV, we compare our calculations to one dimensional models to get a more fundamental understanding and a numerically easier access to the superconducting coherence length.

\section{METHOD}

This implementation will rely on the existing real-space screened KKR impurity code [41] in combination with the $\mathrm{BdG}$ solver for the periodic lattices [38]. Here, we will focus on the most relevant aspects crucially relevant for the consideration in real-space impurity systems. All equations are given in Rydberg units. The effective potentials within the theory of superconducting density functional theory (DFT), exploiting the same approximations as highlighted in Ref. [38], are the electron potential $V_{\text {eff }}(\mathbf{r})$ and the effective pairing potential $\Delta_{\text {eff }}(\mathbf{r})$,

$$
\begin{gathered}
V_{\text {eff }}(\mathbf{r})=V_{\text {ext }}(\mathbf{r})+\int d^{3} r \frac{\rho(\mathbf{r})}{\left|\mathbf{r}-\mathbf{r}^{\prime}\right|}+\frac{\delta E_{\mathrm{xc}}[\rho]}{\delta \rho(\mathbf{r})}, \\
\Delta_{\text {eff }}(\mathbf{r})=\Lambda \chi(\mathbf{r}) .
\end{gathered}
$$

Here, $\chi(\mathbf{r})$ is the anomalous density, $\Lambda$ is the interaction parameter and $E_{\mathrm{xc}}[\rho]$ is the exchange correlation functional for the normal state. All densities are expressed via the Green's function

$$
\begin{aligned}
\rho(\mathbf{r})= & -\frac{1}{\pi} \int_{-\infty}^{\infty} d \epsilon f(\epsilon) \operatorname{Im} \operatorname{Tr} G^{e e}\left(\epsilon, \mathbf{r}, \mathbf{r}^{\prime}\right) \\
& -\frac{1}{\pi} \int_{-\infty}^{\infty} d \epsilon[1-f(\epsilon)] \operatorname{Im} \operatorname{Tr} G^{h h}\left(\epsilon, \mathbf{r}, \mathbf{r}^{\prime}\right), \\
\chi(\mathbf{r})=- & \frac{1}{4 \pi} \int_{-\infty}^{\infty} d \epsilon[1-2 f(\epsilon)] \operatorname{Im} \operatorname{Tr} G^{e h}\left(\epsilon, \mathbf{r}, \mathbf{r}^{\prime}\right) \\
- & \frac{1}{4 \pi} \int_{-\infty}^{\infty} d \epsilon[1-2 f(\epsilon)] \operatorname{Im} \operatorname{Tr} G^{h e}\left(\epsilon, \mathbf{r}, \mathbf{r}^{\prime}\right)
\end{aligned}
$$

where the Bogoliubov-de Gennes Hamiltonian $\hat{H}_{\mathrm{BdG}}(\mathbf{r})$ and Green's function $\hat{G}_{\mathrm{BdG}}(z)$ are defined as

$$
\hat{G}_{\mathrm{BdG}}(z)=\left(\begin{array}{ll}
\hat{G}^{e e}(z) & \hat{G}^{e h}(z) \\
\hat{G}^{h e}(z) & \hat{G}^{h h}(z)
\end{array}\right)=\left(z \hat{I}-\hat{H}_{\mathrm{BdG}}\right)^{-1},
$$

with $\hat{H}_{\mathrm{BdG}}(\mathbf{r})=\left\langle\mathbf{r}\left|\hat{H}_{\mathrm{BdG}}\right| \mathbf{r}\right\rangle$ and

$$
\begin{gathered}
\hat{H}_{\mathrm{BdG}}(\mathbf{r})=\left(\begin{array}{cc}
\hat{H}(\mathbf{r}) & \Delta_{\text {eff }}(\mathbf{r}) \\
\Delta_{\text {eff }}(\mathbf{r})^{*} & -\hat{H}(\mathbf{r})^{*}
\end{array}\right), \\
\hat{H}(\mathbf{r})=-\nabla^{2}+V_{\text {eff }}(\mathbf{r})-\mu .
\end{gathered}
$$

Here, $\mu$ is the chemical potential, $z=\epsilon+i \delta$ and the positive limit is taken such that $\delta \rightarrow 0^{+}$. The impurity system is solved via a Dyson equation,

$$
\hat{G}_{\mathrm{BdG}}^{\mathrm{imp}}(z)=\hat{G}_{\mathrm{BdG}}(z)+\hat{G}_{\mathrm{BdG}}(z)\left(\begin{array}{cc}
\delta \hat{V} & \delta \hat{\Delta} \\
\delta \hat{\Delta}^{*} & -\delta \hat{V}^{*}
\end{array}\right) \hat{G}_{\mathrm{BdG}}^{\mathrm{imp}}(z),
$$

where the potentials are $\delta \hat{V}=\hat{V}_{\text {imp }}-\hat{V}_{\text {bulk }}$ and $\delta \hat{\Delta}=\hat{\Delta}_{\text {imp }}-$ $\hat{\Delta}_{\text {bulk. }}$. Here, $\hat{G}_{\mathrm{BdG}}(z)$ is the Green's function of the unperturbed but superconducting crystal and $\hat{G}_{\mathrm{BdG}}^{\text {imp }}(z)$ is the resulting impurity cluster Green's function. The impurity real-space cluster is embedded within the unperturbed superconducting crystal and Eq. (8) is solved self-consistently relaxing the charge and anomalous densities within the finite impurity cluster.

Within the atomic sphere approximation (ASA) each atom $i$ can be associated with an atomic sphere with the radius $r_{i}^{\mathrm{ASA}}$. Thus the potentials $V_{\text {eff }}(\mathbf{r})$ and $\Delta_{\text {eff }}(\mathbf{r})$ can be written in sums

$$
\begin{aligned}
& V_{\mathrm{eff}}(\mathbf{r})=\sum_{i} V_{i}(\mathbf{r}), \\
& \Delta_{\mathrm{eff}}(\mathbf{r})=\sum_{i} \Delta_{i}(\mathbf{r}),
\end{aligned}
$$

with $V_{i}(\mathbf{r})=0$ and $\Delta_{i}(\mathbf{r})=0$ if $|\mathbf{r}| \geqslant r_{i}^{\mathrm{ASA}}$ and Equ. (2) becomes

$$
\Delta_{i}(\mathbf{r})=\Lambda_{i} \chi_{i}(\mathbf{r})
$$

For the self-consistent cycle, we use a semicircle contour in the upper complex energy plane with at least 50 energy points and for the angular momentum cutoff we consider $l_{\max }=3$ including $f$ electrons. The real-space impurity cluster, embedded within the perfect crystal, for which we considered the full charge relaxation contained in all cases 89 atoms.

\section{NIOBIUM WITH IMPURITIES}

As a first test we consider $\mathrm{N}$ impurities in $\mathrm{Nb}$, a conventional impurity in this elemental superconductor. On one hand we aim to analyze the effect of the impurity on the superconducting state in the surrounding $\mathrm{Nb}$. On the other hand we will explore the interplay between the gap anisotropy as discussed in detail in Ref. [38] and the electron scattering off the substitutional impurity. The self-consistent impurity cluster contains 89 atoms where the boundary condition is the perfect superconducting periodic crystal. The central atom is replaced by a substitutional $\mathrm{N}$ impurity, the interaction parameter at the impurity site is $\Lambda_{\text {imp }}=0$ and we relax the normal charge density $\rho(\mathbf{r})$, as well as the anomalous density $\chi(\mathbf{r})$, within 


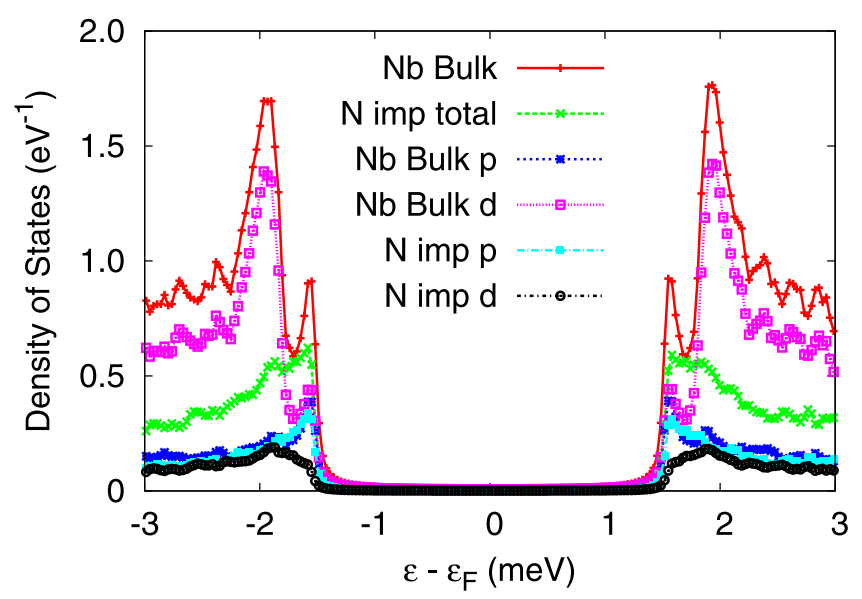

FIG. 1. LDOS ( $s, p, d$, and total) of an $\mathrm{N}$ impurity in comparison to the DOS of unperturbed periodic $\mathrm{Nb}$.

the impurity cluster. The LDOS at the central $\mathrm{N}$ impurity is shown in Fig. 1 and compared to the $\mathrm{Nb}$ DOS of the periodic superconductor. As we set $\Lambda_{\text {imp }}=0$, the quasiparticle gap at the $\mathrm{N}$ site is purely induced from the surrounding superconducting $\mathrm{Nb}$. As such it has the same principle size as $\mathrm{Nb}$ with a notable absence of the outer coherence peak. This follows from the lack of $d$ states in $\mathrm{N}$ and the fact that only the inner coherence peak of $\mathrm{Nb}$ has a significant $p$ character. The larger gap, outer coherence peak, in $\mathrm{Nb}$ is almost entirely of $d$ character.

Reversing this argument the $\mathrm{N}$ impurity should induce strong scattering for the $d$ electrons of the surrounding $\mathrm{Nb}$. This effect is highlighted in Fig. 2 where the LDOS of the nearest-neighbor $\mathrm{Nb}$ atom adjacent to the $\mathrm{N}$ impurity is displayed. A clear broadening between the inner and outer coherence peak is visible, indicating the challenge to resolve the gap anisotropy in this elemental superconductor when structural or chemical perturbations are present. As in real materials such perturbations will be inevitable, making it demanding experimentally to clearly resolve gap anisotropies on the relevant energy scales.

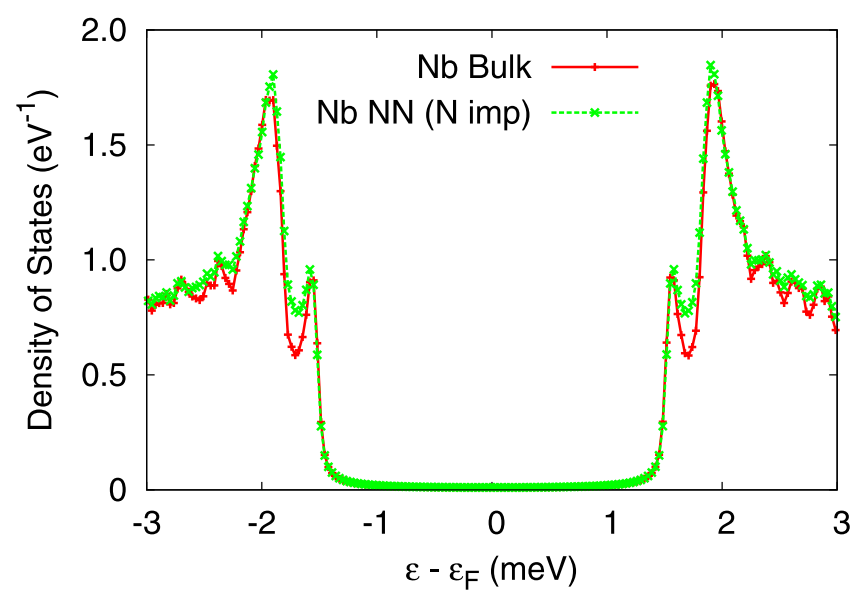

FIG. 2. The DOS of unperturbed periodic $\mathrm{Nb}$ in comparison to the LDOS of the nearest-neighbor $\mathrm{Nb}$ atom atom in the impurity cluster next to $\mathrm{N}$.

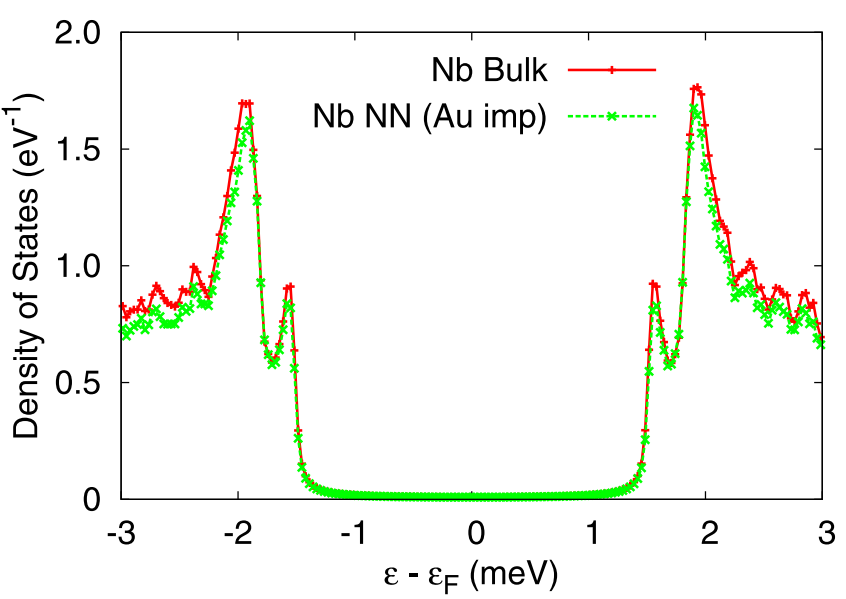

FIG. 3. DOS of unperturbed periodic $\mathrm{Nb}$ in comparison to the LDOS of a nearest-neighbor $\mathrm{Nb}$ atom in the impurity cluster next to the Au impurity.

In order to support our argument that the lack of $d$ orbital character at the $\mathrm{N}$ impurity site is responsible for the effective broadening of the peaks we compare the previous result to a situation where the substitutional impurity is Au contributing significant $d$ character. Figure 3 clearly shows the lack of broadening between the inner and outer coherence peak as the DOS of the unperturbed periodic Nb is compared to the LDOS of the nearest-neighbor $\mathrm{Nb}$ in the impurity cluster containing $\mathrm{Au}$ at its center.

\section{GRANULAR SUPERCONDUCTIVITY}

So far we have analysed the induced superconductivity at the impurity site as well as the impact of the electronic scattering by the impurity atom on the surrounding superconductor. The fact that the superconductor induces a superconducting gap at the impurity site without an effective interaction parameter is not new in principle and has been investigated before $[16,17]$. In the following, we will investigate the inverse problem, where a superconducting impurity cluster is embedded in a nonsuperconducting material. The relevance of this granular superconductivity is its connection to the pseudogap phase of underdoped high- $T_{c}$ cuprate superconductors. In general, superconductivity emerges from two distinct quantum phenomena: pairing between electrons and long range phase coherence. In conventional BCS theory, the condensation of Cooper pairs into a phase-coherent, quantum state takes place simultaneously at the transition temperature. However, in the underdoped high- $T_{c}$ cuprate superconductors the electron pairing occurs at higher temperatures than the long-range phase coherence [42]. In addition, this as been observed in some disordered, amorphous, superconductors [43]. In this model of granular superconductivity the existence of preformed Cooper pairs, pairing without long range phase coherence, are showing similarities with the pseudogap regime of underdoped high- $T_{c}$ cuprate superconductors.

For a relatively small cluster of material with nonzero interaction parameter embedded in a normal metal, the superconductivity will be suppressed and the quasiparticle gap will be forced to close. However, if such a cluster reaches the 


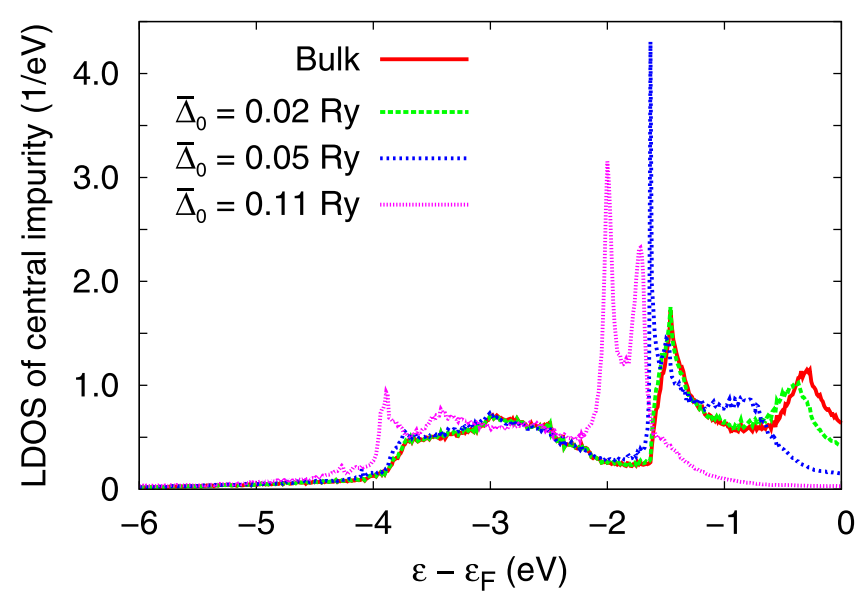

FIG. 4. The LDOS of the central atom in a cluster of 89 atoms with a constant $\Delta_{\text {eff }}$ for every atom in the cluster embedded in metallic $\mathrm{Nb}$ with $\bar{\Delta}_{0}$ defined in Eq. (14).

size of the corresponding superconducting coherence length, $\xi_{0}$, the expectation is that superconductivity can be sustained within the cluster. Within BCS theory [44] the coherence length is given by

$$
\xi_{0}=\frac{\hbar v_{F}}{\pi \Delta},
$$

where $v_{F}$ is the Fermi velocity, linking the coherence length to the inverse size of the superconducting gap $\Delta$ [45]. The coherence length of bulk $\mathrm{Nb}$ is approximately $38 \mathrm{~nm}$ [46]. A cluster of that size would roughly contain $10^{6}$ atoms and is beyond any capability of our method. Within our method, cluster of a few hundred atoms could be considered, limiting the cluster size to $<2 \mathrm{~nm}$. However, it is still possible to test the relation $\xi_{0} \sim \Delta^{-1}$ for artificially enlarged superconducting gaps. In the following, we restricted all calculations to a cluster containing 89 atoms implying a impurity cluster radius of $0.72 \mathrm{~nm}$.

The cluster was constructed from Niobium atoms with a nonzero interaction parameter $\Lambda_{i}$, embedded in an infinite normal state $\mathrm{Nb}$ crystal. In a first step we omit self-consistency and explore the resulting superconducting gap in LDOS calculations when a constant pairing potential $\Delta_{\text {eff }}(\mathbf{r})=\Delta_{\text {eff }}$ is applied. Figure 4 shows the LDOS of the central atom as we change the constant $\Delta_{\text {eff }}$. For a pairing potential of $\Delta_{\text {eff }}=$ $0.11 \mathrm{Ry}$, a gap in the quasiparticle spectrum of approximately $\Delta=1.5 \mathrm{eV}$ is induced, decreasing in size quickly with the size of the pairing potential. At $\Delta_{\text {eff }}=5 \times 10^{-2}$ Ry a suppression of the LDOS is still visible without a full opening of a gap and at $\Delta_{\text {eff }}=2 \times 10^{-2}$ Ry only a small deviation from the nonsuperconducting $\mathrm{Nb}$ remains. This implies that the surrounding metallic $\mathrm{Nb}$ enforces a suppression of the superconducting state as soon as the pairing potential is smaller than $5 \times 10^{-2}$ Ry.

In a next step, we fix the pairing potential at $\Delta_{\text {eff }}=0.11 \mathrm{Ry}$ and explore how the gap in the LDOS develops as we are approaching the boundary to the metallic $\mathrm{Nb}$. In Fig. 5, the corresponding results are summarized, comparing the central atom to the fifth nearest $(0.57 \mathrm{~nm})$ and the seventh nearest $(0.72 \mathrm{~nm})$ shell. Even at a distance of $0.57 \mathrm{~nm}$, the coherence

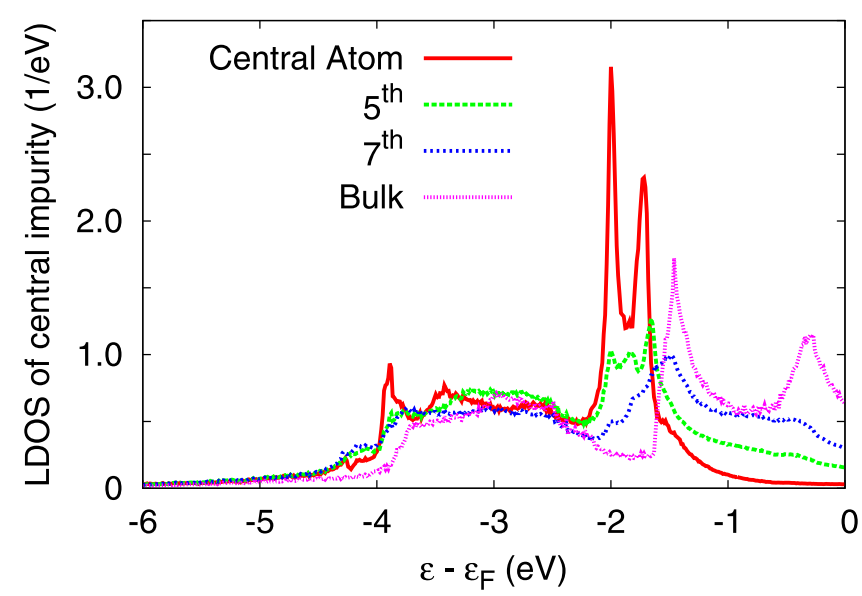

FIG. 5. The LDOS of atoms in the $n$th shell in a cluster of 89 atoms with a constant effective pairing potential $\Delta_{\text {eff }}=0.11 \mathrm{Ry}$, for every atom in the cluster.

peak is still visible but the original gap is fully filled with a slightly suppressed LDOS and the local gap is gradually disappearing. There is no sudden transition from a gapped to a normal state implying the coexistence of anomalous (pairing) as well normal (electron) density.

This finding is very similar to the situation where we change the size of the region within the cluster for which we consider a nonzero and constant pairing potential. The resulting LDOS for the central atom is shown in Fig. 6. Again the coherence peak is more or less visible down to a region of nearest neighbors only but the LDOS at the Fermi energy increases as the cluster is decreasing.

To summarize those findings we define the anomalous charge $\bar{\chi}_{i}$,

$$
\bar{\chi}_{i}=\frac{1}{V_{\mathrm{WS}}} \int_{0}^{r_{\mathrm{ASA}}} d^{3} r \chi_{i}(\mathbf{r}),
$$

which is a constant for each shell at a given distance from the central atom within the cluster. Correspondingly, we define

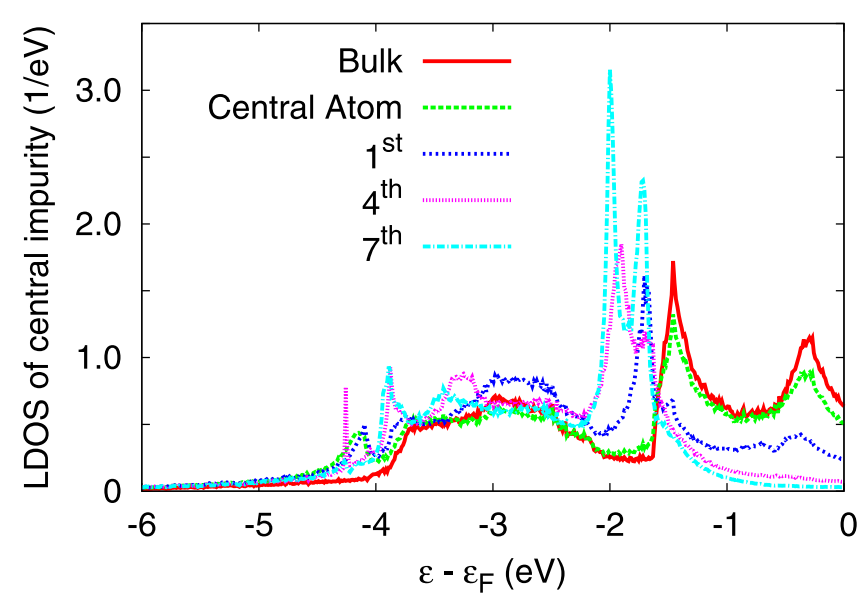

FIG. 6. The LDOS of the central atom in the cluster of 89 atoms with a constant pairing potential $\Delta_{\text {eff }}=0.11$ Ry for every atom up to and including the atoms in the $n$th shell. 


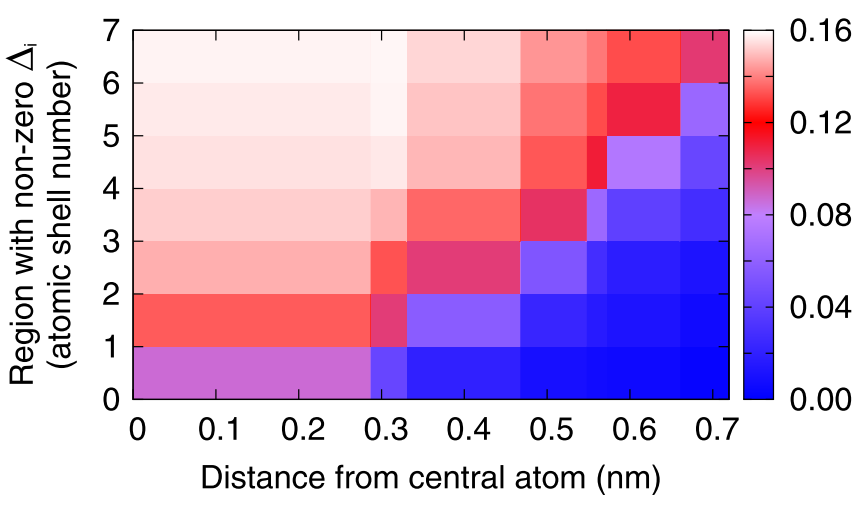

FIG. 7. The average anomalous charge $\bar{\chi}_{i}$ per atom as a function of distance in the cluster for the one-shot gap calculations corresponding to Fig. 6. The $y$ axis represents the region with nonzero constant pairing potential $\Delta_{\text {eff }}=0.11$ Ry up to and including the $n$th shell. The relations between the shell indexes and distances are the following: first: $0.29 \mathrm{~nm}$; second: $0.33 \mathrm{~nm}$; third: $0.47 \mathrm{~nm}$; forth: $0.55 \mathrm{~nm}$; fifth: $0.57 \mathrm{~nm}$; sixth: $0.66 \mathrm{~nm}$; and seventh: $0.72 \mathrm{~nm}$.

the average gap $\bar{\Delta}$,

$$
\bar{\Delta}_{i}=\frac{1}{V_{\mathrm{WS}}} \int_{0}^{r_{\mathrm{ASA}}} d^{3} r \Delta_{i}(\mathbf{r})
$$

which in the self-consistent calculations is related to $\bar{\chi}_{i}$ by the proportionality $\Lambda_{i}$. However, in non-self-consistent one shot calculations the relation is more complex. In Fig. 7, we summarize the results for the anomalous charge $\bar{\chi}_{i}$ as we change the region of nonzero $\bar{\Delta}_{i}(y$ axis in Fig. 7) corresponding to Fig. 6, while at the same time analyzing the full cluster ( $x$ axis in Fig. 7). In all cases, the anomalous charge is quickly reduced if we consider atoms outside the region of the applied nonzero $\bar{\Delta}_{i}$. Nevertheless, it is clearly visible how the anomalous charge is enhanced at the central atom as the region of nonzero $\bar{\Delta}_{i}$ is increased, while at the same time a small anomalous charge is induced beyond the region of nonzero pairing potential.

In order to make a direct connection to the coherence length and its relation to the superconducting gap, it is important to perform all calculations self-consistently. According to the BCS result the cluster needs to be larger than the coherence length to support superconductivity. The complication arises from the fact that we observe pairing (anomalous charge in Fig. 7) while no gap is induced in the quasiparticle spectrum (LDOS, see Fig. 6). In Fig. 8, we summarize the fully selfconsistent calculations changing the constant interaction $\Lambda_{i}$ for the full cluster of 89 atoms. Shown is the LDOS of the central atom. Similarly to our discussion before, the gap in the LDOS vanishes as we reduce the interaction parameter to $\Lambda_{i}=0.3 \mathrm{Ry}$, while the corresponding average gap and thus the pairing potential stays nonzero as highlighted in the legend.

The equivalent summary for the self-consistent calculations to Fig. 7 in case of the one-shot is shown in Fig. 9. A much sharper transition between a vanishing gap is visible in case of a cluster with an applied interaction up to the second shell only.

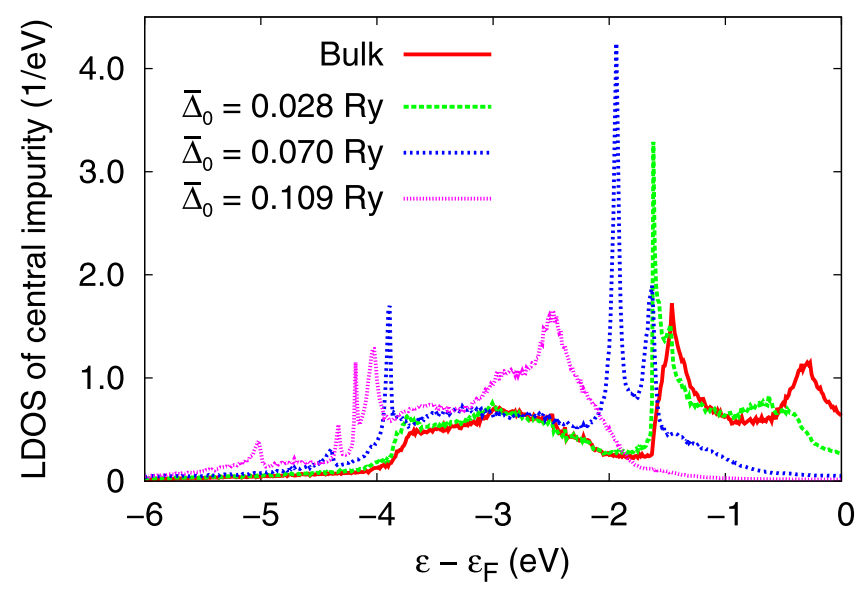

FIG. 8. The LDOS of the central atom in the cluster of 89 atoms with a constant $\Lambda_{i}$ applied to every atom in the cluster. The legend specifies the size of $\bar{\Delta}_{0}$ of the central atom. For the green line $\Lambda=$ $0.3 \mathrm{Ry}$, blue $\Lambda=0.4 \mathrm{Ry}$, and pink $\Lambda=0.5 \mathrm{Ry}$.

In order to better understand the relationship between self-consistency and one-shot LDOS calculations, we have to analyze the relationships between the anomalous charge, $\bar{\chi}_{i}$, the average gap, $\bar{\Delta}_{i}$ and the LDOS at $\epsilon_{F}, D_{i}\left(\epsilon_{F}\right)$. In Fig. 10, $D_{0}\left(\epsilon_{F}\right)$ as a function of $\bar{\Delta}_{0}$ is shown for the central atom in a cluster of 89 atoms. For the self-consistent calculation, a nonzero and constant $\Lambda_{i}$ is applied to all of the atoms up to the seventh nearest-neighbor shell. For the one-shot calculations, a constant pairing potential with the corresponding average gap is applied to all atoms. In this representation, both approaches give very similar results. In all these cases, we observe a smooth transition between the opening of a gap in the quasiparticle spectrum gradually closing in as we change the superconducting strength, either via the interaction parameter, the average applied gap or the size of the superconducting region.

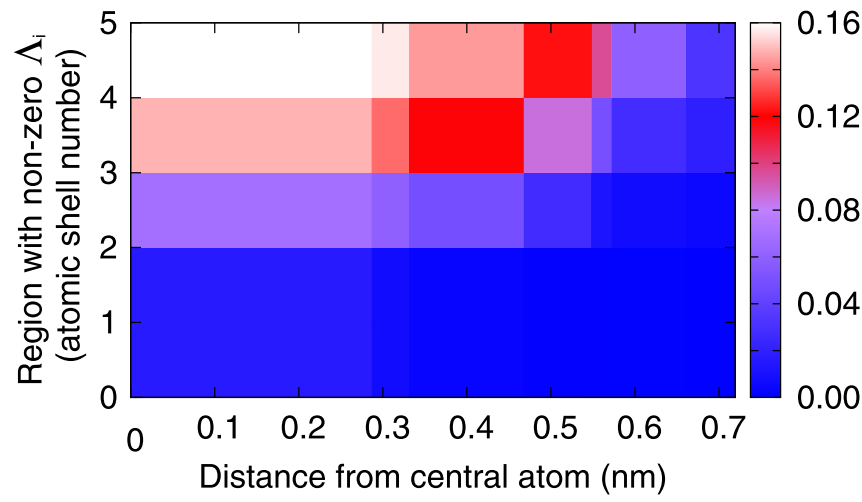

FIG. 9. The average anomalous charge $\bar{\chi}_{i}$ per atom as a function of distance in the cluster. This is the corresponding figure to Fig. 7 but for a fully self-consistent calculation. The $y$ axis represents the region with nonzero coupling parameter $\Lambda_{i}=0.4 \mathrm{Ry}$ up to and including the nth shell. The relations between the shell indexes and distances are the following: first: $0.29 \mathrm{~nm}$; second: $0.33 \mathrm{~nm}$; third: $0.47 \mathrm{~nm}$; fourth: $0.55 \mathrm{~nm}$; fifth: $0.57 \mathrm{~nm}$; sixth: $0.66 \mathrm{~nm}$; and seventh: $0.72 \mathrm{~nm}$. 


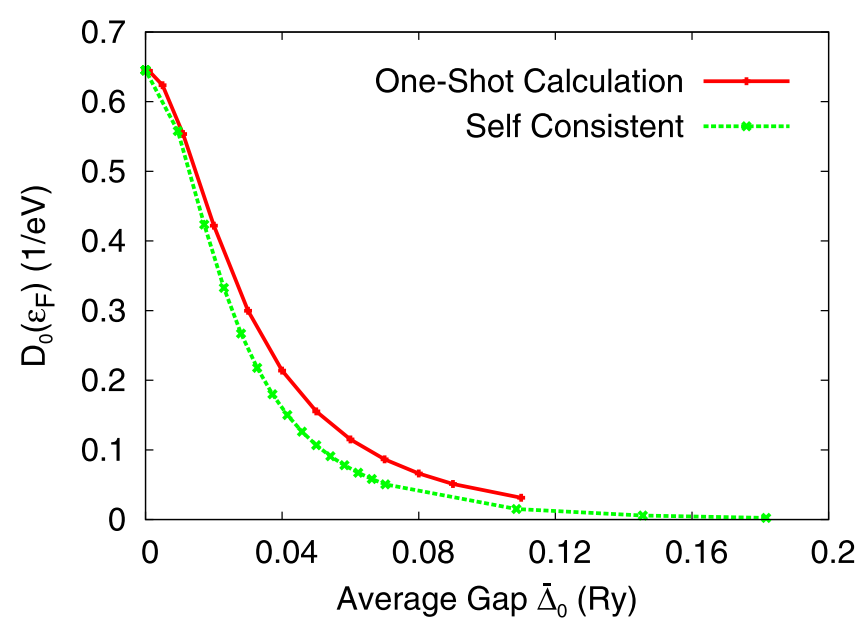

FIG. 10. The LDOS at the Fermi level as a function of the average gap $\bar{\Delta}_{0}$ for the central atom.

However, according to BCS theory, there should be a sharp transition where a gap is induced once the coherence length is reached. This sharp transition becomes visible as we show $D_{0}\left(\epsilon_{F}\right)$ as a function of $\Lambda_{i}$ applied to a cluster of 89 atoms in Fig. 11. This representation highlights the differences between the one shot and the fully self-consistent calculations. Only for the self-consistent calculations we are a able to observe the sharp transition at which the system becomes superconducting at a nonzero interaction parameter. For the one-shot calculations as we reduce the applied average gap we will in all cases observe a nonzero induced anomalous density and as such an effective interaction parameter. In contrast for the selfconsistent calculation, as we reduce the interaction parameter, we eventually reach the point where all superconductivity is suppressed, the anomalous density goes to zero, the gap in the quasiparticle spectrum vanishes and we observe a phase transition. However, while at this point Cooper pairs start to form we do not observe full phase coherence why we do not

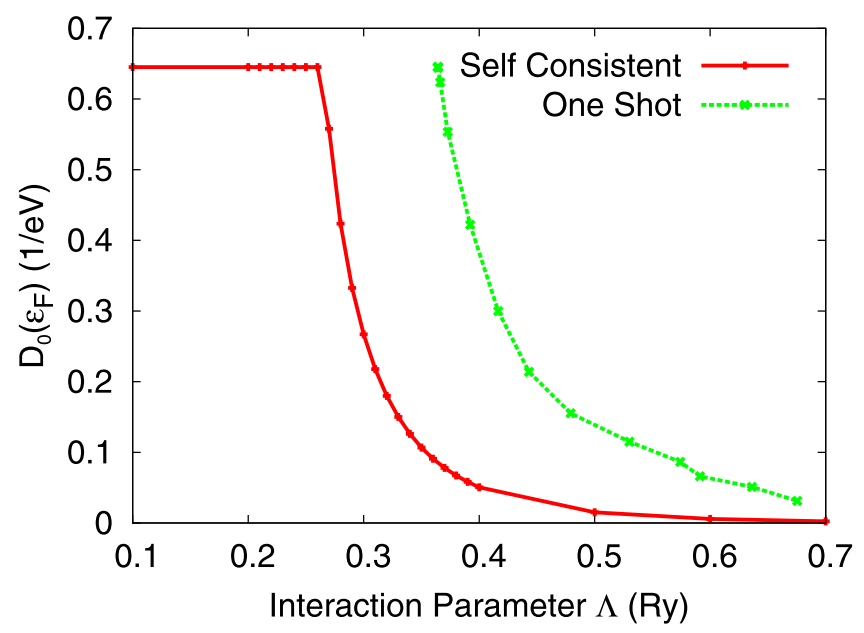

FIG. 11. The LDOS for the central atom at the Fermi level as a function of the interaction parameter. In case of the one-shot calculations, the ratio between the applied average pairing potential and the anomalous charge is used to define an effective interaction parameter.

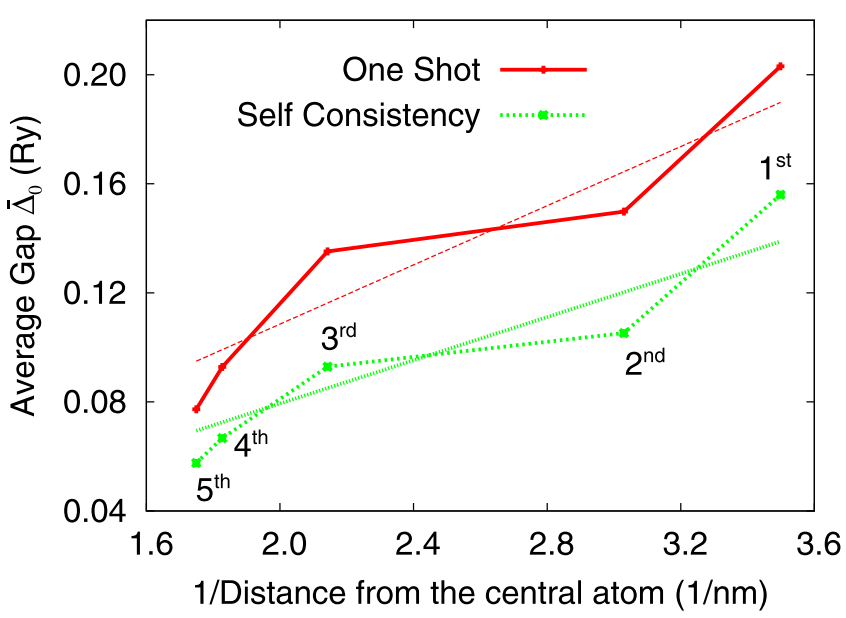

FIG. 12. The average gap $\bar{\Delta}$ as a function of the inverse radius of the region of superconducting atoms. Indicated in the graph are the shell indices corresponding to the distances from the central atom.

observe the opening of a gap in the LDOS as discussed earlier (see Fig. 8).

To finally investigate the coherence length within our method we show in Fig. 12 the average gap as a function of the size of the region with a nonzero interaction parameter. In order to generate this figure, we consider both cases the one shot and the self-consistent calculations. In case of the one shot calculations at a given cluster size we increase the average gap, $\bar{\Delta}_{i}$, until the DOS at the Fermi energy is suppressed below $0.1(\mathrm{eV})^{-1}$. For the self-consistent calculations, we do the same but varying the interaction parameter $\left(\Lambda_{i}\right)$ until we reach the same threshold. The chosen threshold is a trade off between numerical accuracy and reaching a fully gapped situation. According to the BCS relation, Eq. (12), we should find $\bar{\Delta} \propto 1 / \xi_{0}$ with the slope given by $\frac{\hbar v_{F}}{\pi}$. We find a roughly linear dependence with the linear fit giving the slope and as such the Fermi velocity to be $3.52 \times 10^{6}$ and $2.58 \times 10^{6} \mathrm{~m} / \mathrm{s}$ for the one shot and the self-consistent calculations, respectively. This is in reasonable agreement to typical Fermi velocities of the order of $1 \times 10^{6} \mathrm{~m} / \mathrm{s}$.

\section{1D CHAIN RESULTS}

In the previous section, the formation of the full gap in the DOS as a function of the cluster size is somewhat complicated by the limited number of atoms which can be treated in a fully $a b$ initio calculation. Here, we performed a very similar calculation, but on a much simpler system, the 1D chain of periodic, uniform square well potentials. In this model, a finite chain of square well potentials is embedded into an infinite chain of slightly different square well potentials. A certain advantage of the KKR and BdG-KKR theory is, that it can be formulated in a formally similar way to its $3 \mathrm{D}$ counterpart described in Sec. II $[47,48]$. It has the advantage that it can be solved for cluster sizes which are practically impossible in a $3 \mathrm{D}$ calculation. To mimic more closely the $3 \mathrm{D}$ system in the superconducting case, we set the effective pairing interaction to zero in the infinite chain and to a finite value in the impurity region. Then the equations are solved numerically for the embedded cluster of various sizes. 


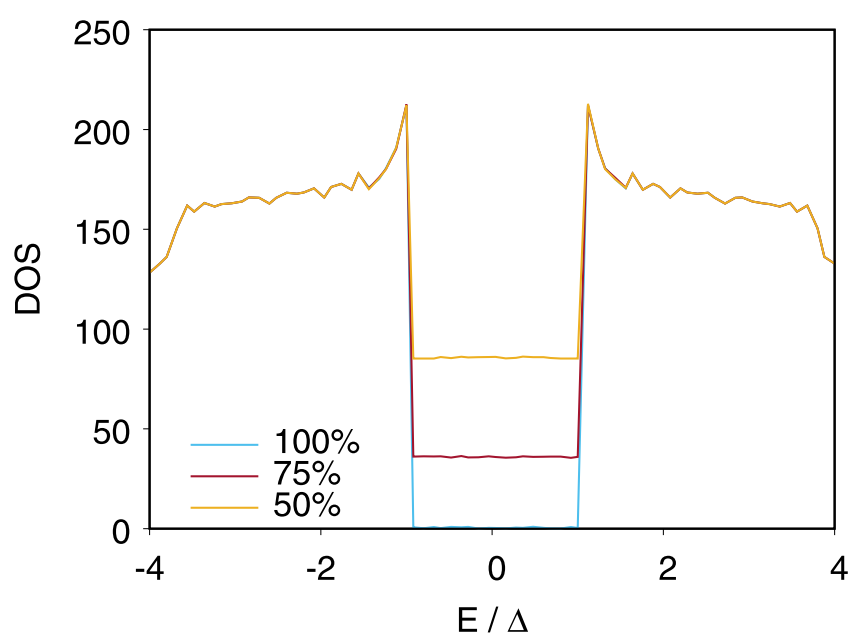

FIG. 13. The local Density of sates (DOS) of the central atom as the chain of superconducting atoms embedded in a metallic systems is increased in length. $100 \%$ refers to the length where there is a full gap. Accordingly shorter lengths are normalized to this.

The analog to Fig. 6 is Fig. 13, considering the LDOS around the Fermi energy of the central atom within the cluster. Evidently, the quasiparticle spectrum does not exhibit a full gap until the size of the cluster reaches a critical length, the coherence length. This is in full analogy to the 3D system shown in Fig. 6. In the same way as before, the LDOS is suppressed around $E_{F}$ for all cluster sizes while the coherence peaks stay rather constant. In this simple $1 \mathrm{D}$ model, the width is equal to the applied effective pairing interaction. To further illustrate this behavior, Fig. 14 shows how the DOS at the Fermi level of the central atom (square well potential) behaves as the size of the impurity chain increases.

Fixing the gap and extracting the chain length for which the LDOS at $E_{f}$ vanishes and repeating this calculation for a range of gaps we summarize these results in Fig. 15. As for the $3 \mathrm{D}$ case this relation should be be compared to Eq. (12). Here the relationship between the gap and the length of the

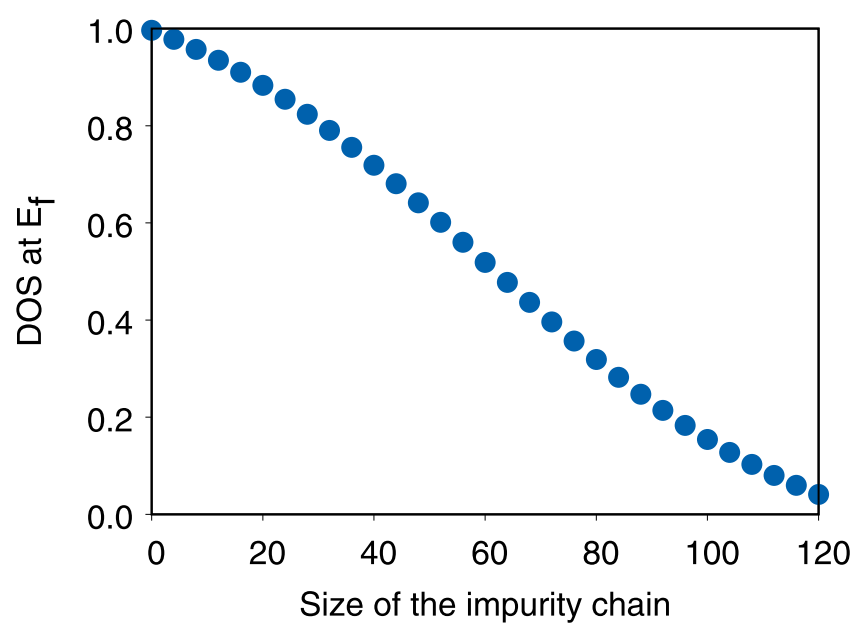

FIG. 14. The LDOS at the Fermi level for the central atom as a function of the chain length (number of square well potentials).

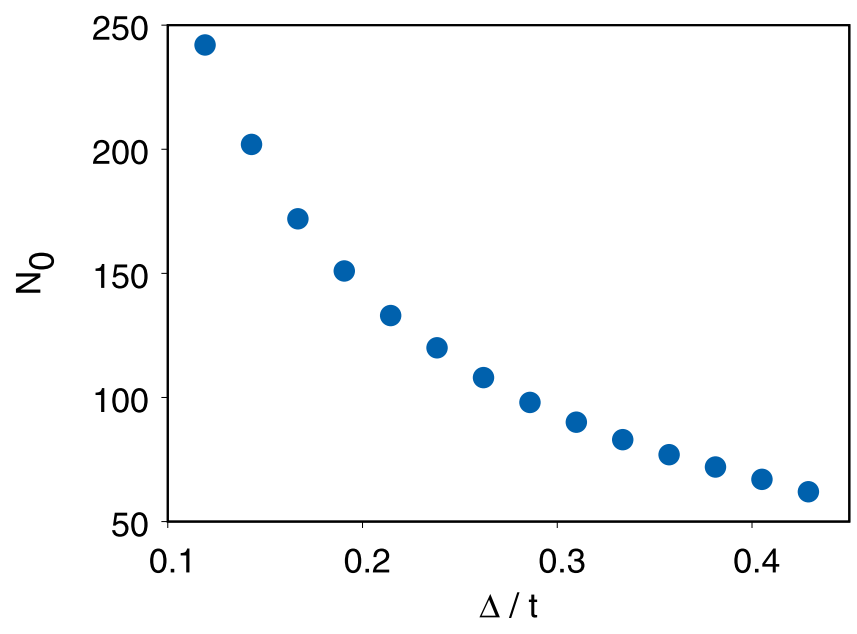

FIG. 15. The minimum cluster size $\left(\mathrm{N}_{0}\right)$ at which a gap can be observed as a function of the effective pairing interaction in the $\mathrm{BdG}$ equations. In this $1 \mathrm{D}$ model, the effective pairing interaction is shown in the units of the single band width $t$.

1D impurity cluster almost perfectly fulfils the prediction of BCS theory. We again may conclude, that the minimum cluster size with a true superconductivity gap is the coherence length.

\section{DISCUSSION AND CONCLUSIONS}

We have implemented a self-consistent solution of the BdG equations into a real-space impurity solver within the KKR formalism, extending the formalism from our previous work [38]. In this formalism both charge, $\rho(\mathbf{r})$ and anomalous $\chi(\mathbf{r})$ densities can be relaxed, with $\Lambda$ being the interaction parameter which drives the superconductivity.

From our previous work, we showed that the gap anisotropy in $\mathrm{Nb}$ is successfully reproduced. Here we show that in the presence of impurities, that gap anisotropy gets broadened by impurity scatterers which contain no 'd' states as that is the main contributor to density of states around the gap. It was not possible to find an impurity which only contained s orbitals at this energy level, potentially obscuring the peaks entirely. However, introducing impurity scattering from $\mathrm{Au}$, an element with $p$ and $d$ character close to the Fermi level no broadening of the peaks at all was observed. This confirmed our argument and underlined the importance of the detailed knowledge of the orbital character of the impurity electrons.

After this we inverted the problem, considering the effect of a nonsuperconducting bulk on a cluster of superconducting impurity atoms. We found that the bulk strongly influences the impurity atoms, similar to our previous study. The gap and the corresponding interaction parameter had to be artificially increased by approximately 1000 times in order to induce a gap within an 89 atom cluster. This is directly related to the superconducting coherence length of the superconducting material. We showed that we were able to reproduce the BCS expression of the coherence length as a function of the superconducting gap. However, we would like to highlight that we clearly observe distinct states of our system. Below a certain 
threshold the interaction is too weak and superconductivity is suppressed throughout the entire system. Passing a critical value we observe the formation of Cooper pairs without full phase coherence leading to a suppression of the LDOS at the Fermi energy without the formation of a full energy gap. Only upon increasing the interaction parameter further phase coherence across the system is achieved and a full gap opens.

To solidify this claim, we perform a simplified $1 \mathrm{D}$ chain KKR model. Here we showed that the coherence length obeys the same trend as for the 3D KKR method. However, due to the easier numerical implementation much larger systems could be explored displaying the relation in a much clearer way.

In summary, we have showed that using a fully $a b$ initio method to describe the normal state and a simple phenomenological parametrization to describe the superconducting exchange correlation functional we can describe the effect of impurities on the superconducting state. Even in the presence of impurities, it is still possible to observe the gap anisotropy in $\mathrm{Nb}$ while depending on the orbital character of the impurity atom a significant broadening of the coherence peaks can be observed. In addition, we have applied a direct method to test the coherence length of superconducting materials which is concurrent with BCS theory. Our future aim will be to include magnetism and spin-orbit coupling to look at more exotic phenomena associated with impurities including Yu-Shiba-Rusinov states and the generation of triplet currents.

\section{ACKNOWLEDGMENTS}

This work was carried out using the computational facilities of the Advanced Computing Research Centre, University of Bristol It was financially supported by the Centre for Doctoral Training in Condensed Matter Physics, funded by EPSRC, Grant No. EP/L015544/1. B.U. was supported by the Hungarian National Research, Development and Innovation Office under Contract No. OTKA K131938. The research reported in this paper and carried out at BME has been supported by the NRDI Fund (TKP2020 IES, Grant No. BME-IE-NAT) based on the charter of bolster issued by the NRDI Office under the auspices of the Ministry for Innovation and Technology. G.C. gratefully acknowledges support from the European Union's Horizon 2020 research and innovation programme under the Marie-Sklodowska-Curie Grant Agreement No. 754510. This work was supported by Spanish MINECO (the Severo Ochoa Centers of Excellence Program under Grant No. SEV-2017-0706), Spanish MICIU, AEI and EUFEDER (Grant No. PGC2018-096955-B-C43), and Generalitat de Catalunya (Grant No. 2017SGR1506 and the CERCA Program). The work was also supported by the European Union MaX Center of Excellence (EU-H2020 Grant No. 824143). The authors would like to thank M. Czerner and Prof. Heiliger for fruitful discussions on the KKR method. In addition, thanks to Ming-Hung Wu and Reena Gupta for many helpful discussions related to the project.
[1] J. Li, M. Ji, T. Schwarz, X. Ke, G. Van Tendeloo, J. Yuan, P. J. Pereira, Y. Huang, G. Zhang, H. L. Feng, Y. H. Yuan, T. Hatano, R. Kleiner, D. Koelle, L. F. Chibotaru, K. Yamaura, H. B. Wang, P. H. Wu, E. Takayama-Muromachi, J. Vanacken, and V. V. Moshchalkov, Nat. Commun. 6, 7614 (2015).

[2] H. Alloul, J. Bobroff, M. Gabay, and P. J. Hirschfeld, Rev. Mod. Phys. 81, 45 (2009).

[3] A. M. Stacy, J. V. Badding, M. J. Geselbracht, W. K. Ham, G. F. Holland, R. L. Hoskins, S. W. Keller, C. F. Millikan, and H. C. Zur Loye, J. Am. Chem. Soc. 109, 2528 (1987).

[4] M. K. Wu, J. R. Ashburn, C. J. Torng, P. H. Hor, R. L. Meng, L. Gao, Z. J. Huang, Y. Q. Wang, and C. W. Chu, Phys. Rev. Lett. 58, 908 (1987).

[5] Y. Enomoto, T. Murakami, M. Suzuki, and K. Moriwaki, Jpn. J. Appl. Phys. 26, L1248 (1987).

[6] J. S. Van Dyke, J. C. Séamus Davis, and D. K. Morr, Phys. Rev. B 93, 041107(R) (2016).

[7] P. O. Sprau, A. Kostin, A. Kreisel, A. E. Böhmer, V. Taufour, P. C. Canfield, S. Mukherjee, P. J. Hirschfeld, B. M. Andersen, and J. C. S. Davis, Science 357, 75 (2017).

[8] A. Kreisel, B. M. Andersen, P. O. Sprau, A. Kostin, J. C. Séamus Davis, and P. J. Hirschfeld, Phys. Rev. B 95, 174504 (2017).

[9] A. Kostin, P. O. Sprau, A. Kreisel, Y. X. Chong, A. E. Böhmer, P. C. Canfield, P. J. Hirschfeld, B. M. Andersen, and J. C. Davis, Nat. Mater. 17, 869 (2018).

[10] N. Hatter, B. W. Heinrich, D. Rolf, and K. J. Franke, Nat. Commun. 8, 8988 (2017).
[11] M. Ruby, B. W. Heinrich, Y. Peng, F. von Oppen, and K. J. Franke, Phys. Rev. Lett. 120, 156803 (2018).

[12] B. W. Heinrich, J. I. Pascual, and K. J. Franke, Prog. Surf. Sci. 93, 1 (2018).

[13] S. Kezilebieke, M. Dvorak, T. Ojanen, and P. Liljeroth, Nano Lett. 18, 2311 (2018).

[14] G. C. Ménard, C. Brun, R. Leriche, M. Trif, F. Debontridder, D. Demaille, D. Roditchev, P. Simon, and T. Cren, Eur. Phys. J.: Spec. Top. 227, 2303 (2019).

[15] G. Deutscher, The Physics of Superconductors (SpringerVerlag, Berlin, 2004), pp. 25-53.

[16] A. L. Fetter, Phys. Rev. 140, A1921 (1965).

[17] M. E. Flatté and J. M. Byers, Phys. Rev. B 56, 11213 (1997).

[18] Q.-H. Wang and D.-H. Lee, Phys. Rev. B 67, 020511(R) (2003).

[19] T. Pereg-Barnea and M. Franz, Phys. Rev. B 78, 020509(R) (2008).

[20] Y. Y. Zhang, C. Fang, X. Zhou, K. Seo, W. F. Tsai, B. A. Bernevig, and J. Hu, Phys. Rev. B 80, 094528 (2009).

[21] P. J. Hirschfeld, M. M. Korshunov, and I. I. Mazin, Rep. Prog. Phys. 74, 124508 (2011).

[22] P. J. Hirschfeld, D. Altenfeld, I. Eremin, and I. I. Mazin, Phys. Rev. B 92, 184513 (2015).

[23] M. A. Sulangi, M. P. Allan, and J. Zaanen, Phys. Rev. B 96, 134507 (2017).

[24] J. E. Hoffman, K. McElroy, D.-H. Lee, K. M. Lang, H. Eisaki, S. Uchida, and J. C. Davis, Science 297, 1148 (2002).

[25] K. McElroy, R. W. Simmonds, J. E. Hoffman, D. H. Lee, J. Orenstein, H. Eisaki, S. Uchida, and J. C. Davis, Nature (London) 422, 592 (2003). 
[26] Ø. Fischer, M. Kugler, I. Maggio-Aprile, C. Berthod, and C. Renner, Rev. Mod. Phys. 79, 353 (2007).

[27] S. H. Ji, T. Zhang, Y. S. Fu, X. Chen, X. C. Ma, J. Li, W. H. Duan, J. F. Jia, and Q. K. Xue, Phys. Rev. Lett. 100, 226801 (2008).

[28] T. Hanaguri, S. Niitaka, K. Kuroki, and H. Takagi, Science 328, 474 (2010).

[29] M. P. Allen, A. W. Rost, A. P. Mackenzie, Y. Xie, J. C. Davis, K. Kihou, C. H. Lee, A. Iyo, H. Eisaki, and T.-M. Chuang, Science 336, 563 (2012).

[30] M. P. Allan, F. Massee, D. K. Morr, J. Van Dyke, A. W. Rost, A. P. Mackenzie, C. Petrovic, and J. C. Davis, Nat. Phys. 9, 468 (2013).

[31] B. B. Zhou, S. Misra, E. H. Da Silva Neto, P. Aynajian, R. E. Baumbach, J. D. Thompson, E. D. Bauer, and A. Yazdani, Nat. Phys. 9, 474 (2013).

[32] Y. Ronen, Y. Cohen, J. H. Kang, A. Haim, M. T. Rieder, M. Heiblum, D. Mahalu, and H. Shtrikman, Proc. Natl. Acad. Sci. U.S.A. 113, 1743 (2016).

[33] N. Avraham, J. Reiner, A. Kumar-Nayak, N. Morali, R. Batabyal, B. Yan, and H. Beidenkopf, Adv. Mater. 30, 1707628 (2018).

[34] F. Giustino, Rev. Mod. Phys. 89, 015003 (2017).

[35] D. J. Choi, C. Rubio-Verdú, J. De Bruijckere, M. M. Ugeda, N. Lorente, and J. I. Pascual, Nat. Commun. 8, 15175 (2017).

[36] H. Ebert, D. Ködderitzsch, and J. Minár, Rep. Prog. Phys. 74, 096501 (2011).

[37] G. Csire, B. Újfalussy, J. Cserti, and B. Gyorffy, Phys. Rev. B 91, 165142 (2015).
[38] T. G. Saunderson, J. F. Annett, B. Újfalussy, G. Csire, and M. Gradhand, Phys. Rev. B 101, 064510 (2020).

[39] G. Csire, B. Ujfalussy, and J. F. Annett, Eur. Phys. J. B 91, 217 (2018).

[40] S. K. Ghosh, G. Csire, P. Whittlesea, J. F. Annett, M. Gradhand, B. Újfalussy, and J. Quintanilla, Phys. Rev. B 101, 100506(R) (2020).

[41] M. Gradhand, D. V. Fedorov, P. Zahn, and I. Mertig, Phys. Rev. B 81, 020403(R) (2010).

[42] V. J. Emery and S. A. Kivelson, Nature 374, 434 (1995).

[43] T. Dubouchet, B. Sacépé, J. Seidemann, D. Shahar, M. Sanquer, and C. Chapelier, Nat. Phys. 15, 233 (2019).

[44] J. Ketterson and S. N. Song, Superconductivity (Cambridge University Press, Cambrdige, 1999), pp. 195-254.

[45] Although we use a local pairing model it leads to Cooper pairs which are extended in real-space since Cooper pairs are formed in momentum space and not in real space. In fact our local pairing model is the analog of conventional BCS theory, where Cooper pairs are formed by electrons with different quantum numbers $\{\mathbf{k}, \uparrow\}$ and $\{-\mathbf{k}, \downarrow\}$ in which states from the region of the gap around the Fermi level are mixed. The coherence length is the extension of these wave packets in real space, which is only indirectly related to the pairing model.

[46] C. Kittel, Elementary Solid State Physics (Wiley, New York, 2005), p. 276.

[47] M. B. Suvasini and B. L. Gyorffy, Phys. C: Supercond. 195, 109 (1992).

[48] W. H. Butler, Phys. Rev. B 14, 468 (1976). 